\title{
PRAGMÁTICA, MODO Y CONDICIONALES
}

EDUARDO BUSTOS GUADAÑO

Universidad Nacional de

Educación a Distancia, Madrid

\section{Condicionales y actitudes epistémicas}

Nuestra hipótesis general es que ciertas formas modales en que se presenta el antecedente de una oración condicional dependen de la intención de expresar actitudes epistémicas por parte del hablante o, dicho de otro modo, que la modalidad verbal en el antecedente de una oración condicional es un recurso expresivo convencional en castellano para la manifestación de actitudes epistémicas. ${ }^{1}$ La elección de uno u otro modo por parte del hablante, cuando esta elección es posible, se puede explicar en términos de su intención primaria de dar a entender una actitud epistémica y de su intención secundaria de que la intención primaria sea reconocida por la audiencia. Las actitudes epistémicas posibles se inscriben en un continuo epistémico, que va desde la extrema seguridad en la verdad de la cláusula condicional (el hablante sabe $\alpha$ o cree que sabe $\alpha$ ) a la extrema seguridad en su falsedad (el hablante sabe no $\alpha$ o cree que sabe no $\alpha$ ). No obstante, en la explicación de la modalidad verbal de las oraciones condicionales mediante la apelación a los principios conversatorios que regulan su interpretación, juegan un papel más importante los puntos intermedios en el continuo epistémico. Esos puntos se corresponden con las situaciones en que el hablante tiene creencias no muy segu-

1 Por tanto, esta hipótesis es diferente de la avanzada por Hooper y Terrell (1974) de acuerdo con la cual los modos estarían relacionados con el contraste aserción/presuposición a través de las clases semánticas de vervos. A. Manteca (1981) ha indicado las deficiencias de esta hipótesis, entre las cuales no es la menor la de considerar que las nociones de aserción y presuposición son semánticas y que, en consecuencia, están sistemáticamente relacionadas con hechos sintácticos. 
ras sobre el valor veritativo de la cláusula antecedente de la oración condicional y no un auténtico conocimiento de tal valor veritativo.

Esta hipótesis general es susceptible de integración en consideraciones generales sobre la alternancia indicativo/ subjuntivo en español, de tal modo que en realidad constituiría parte de una solución teórica más integral. En última instancia, estarían en juego las creencias epistémicas del hablante y la naturaleza del contexto comunicativo. Estas creencias, la intención del hablante de manifestarlas y de que sean reconocidas por la audiencia (Grice, 1968, 1969), son las que en definitiva explicarían el uso de los modos indicativo y subjuntivo cuando tal utilización no viene impuesta por el sistema de la lengua. Mientras que el uso del modo indicativo abarca una parte del espectro de la manifestación de tales creencias epistémicas, el del subjuntivo abarca uno diferente, aunque en ciertos contextos puedan solaparse. El uso del indicativo está asociado con actitudes epistémicas positivas hacia el valor de verdad de la oración en que aparece, mientras que la neutralidad epistémica, ligada al uso del subjuntivo, incluye actitudes epistémicas que son compatibles tanto con la inseguridad del hablante en el valor de verdad como con la sugerencia o implicatura de que tal valor es el de falsedad.

Para mostrar la forma en que el análisis de las estructuras condicionales se enmarca en el análisis más amplio de la contraposición indicativo/subjuntivo, comenzaremos poniendo de relieve la similaridad existente con un caso de alternancia modal relacionado semánticamente con las estructuras condicionales - la relación consiste en una identidad de estructura lógica. Se trata de las oraciones subordinadas consecutivas, que admiten la alternancia indicativo/subjuntivo: ${ }^{2}$

2 Klein (1975) ha relacionado Ia alternancia indicativo/subjuntivo en estas oraciones con el contraste aserción/no aserción. El hablante, cuando escoge 
(1) Como tienen dinero, podrán esperar otro año.

Este tipo de oraciones se puede usar en español para expresar una relación causal entre los dos hechos expresados por las oraciones subordinada y principal. ${ }^{3}$ Igualmente ésa puede ser la función de algunas oraciones condicionales, siendo éstas prácticamente equivalentes, al menos en cuanto a sus condiciones de verdad, a las oraciones consecutivas: ${ }^{4}$

(2) Si tienen dinero, podrán esperar otro año.

(Obsérvese que la partícula si es incompatible con el presente de subjuntivo.)

Ahora bien, examinemos las posibles interpretaciones de (2) a la luz de las creencias epistémicas que mediante su proferencia puede expresar un hablante de español. En abstracto, al margen de un contexto determinado, las interpretaciones de (2) pueden oscilar entre la que atribuye al hablante el conocimiento o la creencia en la verdad de la prótasis, algo así como

(3) a. Si tienen dinero, y sé que lo tienen, ...

b. Si tienen dinero, y creo que lo tienen, ...

y la interpretación que mantendría al hablante espistémicamente neutral con respecto a tal valor veritativo:

(4) Si tienen dinero, y yo no sé si lo tienen, ...

el indicativo, estaría ejecutando una aserción, mientras que no se realizaría tal acto de habla si elige el subjuntivo. Por las consideraciones que siguen, se comprobará que tal equiparación dista de ser perfecta.

3 De las consideraciones que siguen, se desprende que no siempre es cierto que las oraciones consecutivas en indicativo equivalgan a oraciones causales y las que van en subjuntivo a oraciones condicionales como, al parecer, mantiene Manteca (1981 pp. 82-83).

4 La conexión entre la expresión de causalidad, las oraciones consecutivas y las condicionales, ha sido puesta de relieve por R. Lapesa (1979). 
Ambos tipos de interpretaciones no son disjuntas entre sí, en el sentido de que la que corresponde a (4) es compatible con (3b) y también con su negación: el efecto de la partícula condicionante $s i$ puede ser el de inducir la interpretación de una actitud epistémica neutral por parte del hablante. La oración condicional en indicativo ocupa por tanto un segmento del continuo epistémico de las actitudes del hablante que, en un extremo, la hace equivalente de una oración consecutiva con subordinada en indicativo y, en el otro, con esa oración con cláusula subordinada en subjuntivo. Es decir, la oración condicional en indicativo, en particular cuando expresa una relación causal, es equivalente, desde el punto de vista pragmático, tanto a una oración consecutiva en indicativo como en subjuntivo. Nótese, sin embargo, que quedan fuera de este ámbito interpretativo las actitudes epistémicas no creo que $\alpha$, creo que no $\alpha$ y sé que no $\alpha$, en especial esta última que, como veremos, determina la introducción del modo subjuntivo.

\section{La interpretación de las oraciones condicionales}

¿Qué principio guía a la audiencia en la interpretación de las oraciones condicionales en indicativo? ¿Cuáles son los criterios que sigue tal audiencia en la elección de una $u$ otra interpretación? Como las oraciones condicionales con antecedente en indicativo son compatibles con la expresión de diferentes actitudes epistémicas, los principios que permiten inferir a una audiencia tales actitudes han de ser de naturaleza contextual. La regla es bien sencilla en un caso: la audiencia atribuirá al hablante la actitud epistémica sé que $\alpha$ (o creo que $\alpha$ ), ante la proferencia de una oración con estructura lógica $\alpha \rightarrow \beta$ (con $\alpha$ en indicativo), si $\alpha$ forma parte del contexto anterior a dicha proferencia. Pero si $\alpha$ no forma parte de tal contexto, la interpretación "preferible" para la audiencia es la que asigna una actitud episté- 
mica neutral al hablante. El funcionamiento de estos principios interpretativos queda más claro en un ejemplo:
(5) $\mathrm{H}_{1}$.-A los Pérez les ha tocado la lotería.
$\mathrm{H}_{2}$. - Entonces tienen bastante dinero...
$\mathrm{H}_{1}$.-Así es. Y, si tienen dinero, podrán esperar otro año.

En este intercambio comunicativo, la creencia en la verdad del hecho enunciado por la prótasis ha sido introducida previamente en el contex̧to; tanto $\mathrm{H}_{1}$ como $\mathrm{H}_{2}$ comparten esa creencia, de tal modo que pertenece a la base común del contexto (Bustos, 1982). En consecuencia, la interpretación de la oración condicional que asigna a $\mathrm{H}_{1}$ una actitud epistémica neutral queda automáticamente excluida (en aplicación de la Máxima conversatoria de Cantidad; Grice, 1970). La oración condicional expresa una relación causal entre un hecho conocido y aceptado y un (posible) hecho futuro. En cambio, en la conversación

(6) $\mathrm{H}_{1}$. - Si los Pérez tienen dinero, podrán esperar otro año. $\mathrm{H}_{2}$.-Pero, ¿tienen dinero en realidad?

$\mathrm{H}_{1}$.-Pues no estoy seguro, pero creo que sí.

la oración condicional de $\mathrm{H}_{1}$ es susceptible de una interpretación que asigna a éste una actitud epistémica neutral, pues $\alpha$ no forma parte del contexto que manejan hablante y audiencia. La conversación progresa, por parte de la audiencia, $\mathrm{H}_{2}$, en la dirección del logro de la especificación o concreción de tal interpretación. Lo que consigue $\mathrm{H}_{2}$ es que $\mathrm{H}_{1}$ formule de un modo concreto la actitud epistémica que mantiene y que es compatible con la interpretación que equipara la oración condicional a una oración consecutiva en subjuntivo. Pero las actitudes epistémicas creo que no $\alpha$ y no creo que $\alpha$ son también compatibles con el uso del indicativo en la prótasis de las oraciones condicionales, 
es decir, que en (6) $H_{1}$ podría haber respondido a la pregunta de $\mathrm{H}_{2}$ diciendo:

(7) a. Pues no estoy seguro, pero creo que no. b. Pues no estoy seguro, pero no creo.

No obstante, hay que subrayar que tal interpretación es menos frecuente, puesto que el uso del indicativo en estructuras condicionales está en general asociado con hablantes que tienen una buena competencia lingüística, a actitudes epistémicas positivas hacia la prótasis en tal modo.

En resumen, en el caso de oraciones condicionales con antecedente en indicativo, el principio de interpretación preferencial, el principio que permite a la audiencia asignar la actitud epistémica correcta a quien profiere tales oraciones es: el hablante cree que $\alpha$ o sabe que $\alpha$, si $\alpha$ forma parte del contexto de proferencia de $\alpha \rightarrow \beta$. La oración $\alpha$ va consecuentemente en indicativo, porque el em. pleo del subjuntivo excluiría automáticamente la atribución de actitudes epistémicas positivas al hablante. Si $\alpha$ no forma parte del contexto previo a la proferencia de $\alpha \rightarrow \beta$, la audiencia está autorizada a inducir una actitud epistémica neutral en el hablante: no sé si a. Esta actitud epistémica neutral abarca al menos dos puntos en el continuo epistémico que va desde sé que $\alpha$ a sé que no $\alpha$, esto es, es compatible tanto con creo que $\alpha$, como con creo que no $\alpha$, aunque esta última actitud epistémica sea menos frecuente y correcta cuando se profiere una oración condicional con antecedente en indicativo.

\section{Condicionales e implicaturas}

De todo lo dicho hasta ahora hay que concluir que la relación pragmática existente entre la proferencia de una oración condicional y las creencias que la audiencia puede inferir a partir de tal proferencia es de implicatura conversatoria. 
La razón es que existe una estrecha dependencia entre la asignación de una interpretación u otra y el contexto. Por decirlo de otro modo, el hecho de que se asigne al hablante una actitud epistémica u otra depende de que se hallen presentes determinados elementos contextuales que son independientes de la naturaleza convencional del significado de las proferencias. Éste es un aspecto que singulariza el caso de los condicionales con respecto a los demás casos en que se da la alternancia entre los modos indicativo y subjuntivo. En estos casos se puede considerar que el uso del indicativo introduce una implicatura convencional, la manifestación de una creencia epistémica ligada al significado convencional de tal modo. Sin embargo, cuando el indicativo es usado en una prótasis, ese aspecto convencional de su significado queda bloqueado por el uso de la partícula condicionante $s i$, cuyo efecto pragmático general es poner entre paréntesis la (posible) actitud epistémica positiva ligada al uso del indicativo. Es la audiencia, partiendo de la base común contextual, la que determina cuál es de hecho la actitud epistémica del hablante que va ligada a la utilización del indicativo en su caso. Para ello hace uso de su conocimiento del contexto y de los principios generales que representan las máximas conversatorias. Es decir, bajo el supuesto de que la expresión lingüística del hablante es racional, la audiencia asigna a éste la actitud epistémica compatible, por un lado, con su utilización del modo indicativo $y$, por otro, con la naturaleza del contexto, tal como ella lo concibe. Existe pues, en el caso de la estructura condicional indicativa un mayor margen para la interpretación que en el caso de estructuras alternantes indicativo/subjuntivo. La interpretación en estos últimos casos está "determinada" por el significado convencional de los modos indicativo y subjuntivo, mientras que en las oraciones condicionales tal significado está bloqueado por la propia estructura condicional y, en particular, por el uso de la partícula si. El vínculo 
existente entre el uso del indicativo y la interpretación que asigna a su usuario una actitud epistémica positiva es mucho más fuerte, de hecho cuasi-semántico, en el caso de las oraciones con alternancia indicativo/subjuntivo que no son condicionales, que en las de esta clase. En realidad, para la asignación de la actitud epistémica positiva en oraciones con alternancia no es necesario que la audiencia considere la naturaleza del contexto, dado el carácter convencional del uso del indicativo en estas oraciones. En cambio, como hemos visto, la consideración del contexto es determinante cuando la audiencia tiene que asignar una actitud epistémica a un hablante que profiere una oración condicional con antecedente en indicativo. Es ese contexto, tal como la audiencia lo concibe, un polo fundamental en que se asienta la interpretación de tal modo que, sin él, en cuanto estructuras oracionales abstractas, las oraciones condicionales pueden estar sujetas a ambigüedades como la ejemplificada por la oración (2).

\section{La interpretación de los condicionales subjuntivos}

Sobre las oraciones condicionales cuyo antecedente está en subjuntivo se pueden hacer consideraciones parecidas a las hechas respecto a las oraciones condicionales indicativas. La zona del continuo epistémico que cubren tales oraciones va desde $\mathrm{h} \sim K \boldsymbol{\alpha}$ a $\mathrm{h} K \sim \boldsymbol{a}^{5}$. Es decir, cuando un hablante profiere una estructura condicional subjuntiva puede estar expresando una actitud epistémica entre no sé que $\alpha$ y sé que no a. La asignación por parte de la audiencia de una actitud epistémica al hablante dentro de ese continuo depende de factores contextuales similares al caso de las oraciones condicionales con antecedente en indicativo.

En primer lugar, considérese el caso en que la audiencia

- K y C son los operadores epistémicos de conocimiento y creencia respeo tivamente. Se corresponden con los operadores K y B de J. Hintikka (1962) 
puede atribuir al hablante la actitud epistémica $h \sim K \alpha$. Se trata de un caso que las oraciones con prótasis subjuntivas comparten con las indicativas, es decir, un punto de intersección en las convenciones que regulan el uso de la partícula condicionante si en combinación con los modos indicativo y subjuntivo. Podemos utilizar un ejemplo en un contexto en que sea posible tal atribución:

(8) $H_{1}$.-Aún no conozco las calificaciones, pero si Juan Pedro hubiera aprobado, no tendría que volver a examinarse.

$\mathrm{H}_{2}$.-Pues yo sí las conozco y, efectivamente, Juan Pedro ha aprobado.

$\mathrm{H}_{1}$.-Entonces no tiene que volver a examinarse.

En este contexto, típico de un razonamiento contrafáctico, la interpretación que asigna a $\mathrm{H}_{1}$ el desconocimiento del valor de verdad de la prótasis en subjuntivo es más que posible, es casi obligatoria, pues la primera oración de $\mathrm{H}_{1}$ se supone en relación causal con ese desconocimiento. La audiencia atribuye de hecho ese desconocimiento a $\mathrm{H}_{1}$ y orienta su conducta lingüística a su eliminación.

Nótese que no es decisivo el uso del pluscuamperfecto de subjuntivo, puesto que las mismas consideraciones se pueden aplicar a (8) si se cambia la proferencia de $\mathrm{H}_{1}$ a imperfecto de subjuntivo. La diferencia entre 'si Juan Pedro hubiera aprobado' y 'si Juan Pedro aprobara' está en el modo en que subjetivamente concibe el hablante la acción, como (posiblemente) acabada o (posiblemente) en curso de realización.

Ahora bien, bajo la actitud epistémica general $\mathrm{h} \sim \mathrm{K} \alpha$, hay dos actitudes epistémicas concretas compatibles con ella, $\mathrm{hC} \alpha$ y $\mathrm{h} \sim \mathrm{C} \alpha$. En la concreción de estas dos actitudes epistémicas es donde se pueden establecer algunas diferencias entre el uso del indicativo y del subjuntivo. Nuestra tesis, como se puede imaginar, es que el uso del imperfecto o pluscuamperfecto de subjuntivo se encuentra en general 
ligado a la expresión de la actitud epistémica $h \sim C \alpha$ en los contextos habituales de interacción comunicativa, mientras que el uso del indicativo está ligado habitualmente, como hemos indicado, a la expresión de la actitud hCa.

Un contexto en el que evidentemente no vale este intento de generalización es el del razonamiento científico contrafáctico, en que los participantes se suelen abstener, por decirlo así, de mantener prejuicios o actitudes previas injustificadas hacia el valor de verdad de los supuestos a partir de los cuales razonan. Por ejemplo, un matemático que intenta probar que no hay dos conjuntos vacíos diferentes entre sí puede razonar a partir de la consideración de que, si los hubiera, se seguirían tales y cuales consecuencias contradictorias, pero su empleo del condicional contrafáctico no tiene que suponer una creencia negativa previa a su razonamiento. De hecho, puede pensar que es matemáticamente posible lo que piensa demostrar, y justamente ése es el motivo de que razone a partir del supuesto. En realidad, en el razonamiento contrafáctico que se emplea muchas veces en la actividad científica se mantiene una actitud epistémica positiva hacia los supuestos, al contrario de lo que sugiere el empleo, en la manifestación de dicho supuesto en el lenguaje natural, del modo subjuntivo.

Pero, al margen de usos lingüísticos especializados, en la utilización común de la lengua, el empleo del subjuntivo marca, en nuestra opinión, una mayor desconfianza o "descreencia" en la verdad de la prótasis. En este sentido, el contraste entre (9) y (10)

(9) Si Juan Pedro a. hubiera aprobado, ...

(10) Si Juan Pedro a. ha aprobado, ...

b. aprueba, ...

viene marcado por una diferente actitud epistémica por parte de quien profiere uno $u$ otro tipo de oración. No obs- 
tante, sería difícil establecer una conclusión tajante sin un estudio empírico, estadístico, de cómo interpretan dicho contraste los hablantes del español.

Independientemente de que, bajo la atribución de la actitud epistémica $h \sim K \alpha$, la audiencia se incline por $\mathrm{hC} \alpha$ o $h \sim C \alpha$, hay que tener en cuenta el hecho de que el empleo del subjuntivo sirve para la expresión de otras actitudes epistémicas. Dos son fundamentalmente esas actitudes, $\mathrm{hC} \sim \alpha$ y $\mathrm{hK} \sim \alpha$, compatibles entre sí, pues de la segunda se sigue la primera, aunque no a la inversa.

Aunque desde el punto de vista lógico $\mathrm{y} / \mathrm{o}$ sintáctico es difícil establecer una diferencia entre $\mathrm{h} \sim \mathrm{C} \alpha$ y $\mathrm{hC} \sim \alpha$, desde el punto de vista pragmático $\mathrm{y} / \mathrm{o}$ psicológico hay una clara diferencia. Si la audiencia, basándose en su conocimiento del contexto, atribuye al hablante la actitld $\mathrm{h} \sim \mathrm{C} \alpha$, esto no la autoriza a introducir $\sim \alpha$ como una creencia en el contexto, mientras que supone tal introducción la atribución de $\mathrm{hC} \sim \alpha$. Por decirlo de otro modo, $\mathrm{hC} \sim \alpha$ supone una actitud epistémica positiva por parte del hablante, que contribuye directamente al aumento de la consistencia del contexto, mientras que $h \sim \mathrm{C} \propto$ es una actitud epistémica negativa, que contribuye, si lo hace a la consistencia del contexto mediante la eliminación de $a$ como parte de las creencias atribuidas por la audiencia al hablante.

Tres son las situaciones básicas en que la audiencia puede atribuir al hablante la actitud epistémica $\mathrm{hC} \sim \alpha: 1$ ) ante todo, la atribución de $\mathrm{hC} \sim \alpha$ es una consecuencia directa de la atribución epistémica $h K \sim \alpha$. Luego, en todas las ocasiones en que la audiencia esté autorizada a pensar que el hablante sabe la falsedad de $\alpha$, cuando $\alpha$ es la prótasis en subjuntivo de una oración condicional, estará por ello mismo autorizado a la atribución de $\mathrm{hC} \sim \alpha$. La oración queda en éstos inmediatamente incluida en el contexto, si es que no forma parte de él.

2) En segundo lugar, puede que la audiencia, a partir 
del contexto, atribuya al hablante la actitud hCK $\sim a$, es decir, que piense que el hablante cree que sabe la falsedad de la prótasis subjuntiva. Esto sucede cuando la audiencia tiene buenas razones para dudar de tal falsedad, por ejemplo porque sabe que tal falsedad no se da:

(11) $\mathrm{H}_{1}$, -Si Juan Pedro hubiera aprobado, no tendría que volver a examinarse, como tiene que hacerlo.

$\mathrm{H}_{2}$.-Pero ¡si ha aprobado!

$\mathrm{H}_{1}$. -No puede ser, yo mismo miré la lista de las calificaciones.

$\mathrm{H}_{2}$. - Sí, pero se trataba de un error que más tarde fue rectificado.

$\mathrm{La}$ audiencia $\mathrm{H}_{2}$ puede atribuir a $\mathrm{H}_{1}$, a partir de las proferencias de éste, la actitud hCK $\sim \alpha$, de la cual se sigue $\mathrm{hC} \sim a$. Su contribución conversatoria sigue pues la dirección "destructiva" de la eliminación de $\sim \alpha$ del contexto, pues tal creencia es inconsistente con su propio conocimiento de la situación. Es la audiencia la que sabe que $\alpha$ es verdadera, por lo que su objetivo ha de ser hacer entender a $H_{1}$ que la actitud epistémica que mantiene es errónea.

3) En tercer lugar, hay que tener en cuenta que la actitud epistémica $\mathrm{hC} \sim \alpha$ no siempre se sigue de $\mathrm{hCK} \sim \alpha$, sino que puede corresponder a la actitud epistémica $h \sim K \alpha$. Como vimos anteriormente, tal actitud epistémica no sólo es compatible con $h C \alpha$, sino también con $h \sim C \alpha$. Además, también es consistente con la actitud hC $\sim \alpha$, pues un hablante puede reconocer que su creencia $\sim \alpha$ puede ser falsa y seguir manteniéndola. Es decir, no es una condición necesaria para mantener una creencia, $\sim \alpha$ por ejemplo, la creencia en que se sabe $\sim \alpha$. En este sentido, la atribución simultánea de las actitudes epistémicas $\mathrm{h} \sim \mathrm{K} \alpha$ y $\mathrm{hC} \sim \alpha$ es perfectamente consistente y plausible. Por ejemplo:

(12) $\mathrm{H}_{1}$, -Si Juan Pedro hubiera aprobado, podríamos ir jun- 
tos de vacaciones, pero me temo que las tendremos que pasar separados.

$\mathrm{H}_{2}$.-Pero, ; si Juan Pedro ha aprobado!

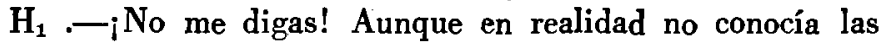
calificaciones, creía que le habían suspendido.

En este caso, $\mathrm{H}_{1}$ tiene una actitud epistémica que claramente es de creencia en la falsedad de la prótasis. Sin embargo, sus expresiones ('me temo', el uso del potencial simple) no permiten atribuirle la creencia en el conocimiento de tal falsedad $(\mathrm{hCK} \sim \alpha)$. De todos modos, el efecto pragmático de introducción de una nueva creencia es el mismo que en (11). La conducta lingüística de la audiencia se orienta pues en el mismo sentido: la eliminación de $\sim \alpha$ como creencia que introduce una inconsistencia en el contexto.

Finalmente, hemos de volver sobre el primer caso, en que la atribución de la actitud hK $\sim \alpha$ implica ya la de hC $\sim \alpha$. Hay que señalar que tal atribución exige como condición la coparticipación, por parte de la audiencia, en la creencia de $\sim \alpha$. En efecto, si la audiencia atribuye al hablante la actitud $\mathrm{hK} \sim \alpha$ es en virtud de una de estas dos razones: o bien la audiencia sabe también $\sim \alpha$, en cuyo caso su creencia $\sim \alpha$ se sigue automáticamente, o bien no lo sabe, pero a través de la proferencia del hablante reconoce su verdad, en cuyo caso también se sigue su creencia en $\sim \alpha$ (e incluso su creencia de que sabe $\sim \alpha$ ).

En el caso de la atribución $h K \sim \alpha, \sim \alpha$ forma parte previa de, o pasa inmediatamente a formar parte de, la base común de creencias que comparten el hablante y la audiencia. Ésta sólo puede atribuir a un hablante el conocimiento de la verdad (o falsedad) de una oración en la medida en que comparte la creencia en cuestión. Sería irracional para un oyente reconocer que un hablante tiene la actitud $\mathrm{hK} \sim \alpha$, y no obstante mantener que $\alpha$ es verda- 
dera, la creencia de que $\alpha$. En el mismo momento en que la audiencia asigne la actitud $\mathrm{hK} \sim \alpha$ al hablante, $\sim \alpha$ entra a formar parte del conocimiento compartido por hablante y audiencia, pero desde el punto de vista de la audiencia. Esta última salvedad es importante, porque pone de relieve de modo particular la gran distancia que separa el punto de vista exterior al intercambio comunicativo del punto de vista interior. En efecto, pueden suceder, en el supuesto de tal atribución $\mathrm{hK} \sim \alpha$ dos cosas: a) que el hablante no tenga tal actitud, esto es, que $h \sim K \sim \alpha$, sino que sencillamente $\mathrm{hC} \sim \alpha$. En este caso, la atribución de la audiencia es errónea, aunque $\sim \alpha$ siga figurando en el conjunto de creencias compartidas, y b) que la atribución epistémica sea errónea desde un punto de vista objetivo, ajeno a las creencias de los participantes en el intercambio comunicativo. Es decir que, aunque $\mathrm{hCK} \sim \alpha$, en realidad $\mathrm{h} \sim \mathrm{K} \sim \alpha$, por ejemplo, porque objetivamente se da $\alpha$. Esta situación se da cuando tanto el hablante como la audiencia están equivocados respecto a la verdad de $\sim \alpha$, aunque no sean conscientes de su error. Sucede que, por una parte, $\alpha$, que oCK $\sim \alpha$ y que $\mathrm{hCK} \sim \alpha$ y que $\mathrm{hCK} \sim \alpha$, formando en consecuencia $\sim \alpha$ parte del contexto que manejan hablante y audiencia. Aunque desde un punto de vista ajeno a la interacción la atribución epistémica es errónea, es operativa, esto es, explica el comportamiento lingüístico (el empleo del modo subjuntivo en este caso) tanto del hablante como el posterior de la audiencia. Lo que en definitiva cuenta para tal explicación son las creencias subjetivas de hablante y audiencia, no si tales creencias son verdaderas o no, están justificadas o no.

El principio de interpretación preferencial con respecto a las oraciones condicionales subjuntivas se puede formular pues del modo siguiente: si h profiere una oración con la estructura $\alpha \rightarrow \beta$, y $\alpha$ está en modo subjuntivo, la audiencia está autorizada a inferir la actitud epistémica $h \sim C \alpha$ si 
$\alpha$ no forma parte del contexto previo, y si $\sim \alpha$ turma parte de la base común compartida por hablante y audiencia, ésta está obligada a inferir la actitud epistémica $\mathrm{hC} \sim a$. Por otro lado, la audiencia está autorizada a asignar la actitud hK $\sim \alpha$, en el caso de la proferencia $\alpha \rightarrow \beta$ en subjuntivo, si $\sim \alpha \varepsilon B_{c}$, esto es si, según tal audiencia, $\sim \alpha$ es parte de la base común de conocimiento compartido por hablante y audiencia.

\section{Conclusión}

En resumen, nuestra tesis sobre lo que se han venido denominando "presuposiciones" de las estructuras condicionales (Lakoff, 1971) pone de relieve que tales presuntas presuposiciones pertenecen en realidad a la clase de fenómenos pragmáticos denominados implicaturas. Lo implicado pragmáticamente en la proferencia de oraciones pertenecientes a tal tipo son actitudes epistémicas por parte del hablante. Las implicaturas son en realidad fruto de dos factores fundamentales en la regulación de cualesquiera situaciones comunicativas. Por una parte, los procedimientos convencionales (el significado convencional) ligados a la expresión de actitudes epistémicas, que en el caso de la alternancia indicativo/subjuntivo en oraciones condicionales están mediados por el uso de la partícula condicionante si. Por otra, la naturaleza del contexto en que se desenvuelven tales interacciones comunicativas, que es determinante, como hemos mantenido en la formulación de los principios de interpretación preferencial, en la asignación de la actitud epistémica correcta por parte de la audiencia a un hablante. En el caso concreto que hemos tratado, la utilización de los modos en estructuras condicionales, resulta evidente que tal uso está ligado a la expresión de actitudes epistémicas que se inscriben en un continuo. Ese continuo va desde el conocimiento del hablante de la verdad de la prótasis, $\mathrm{h} \mathrm{K} \alpha$, hasta el 
conocimiento de su falsedad, hK $\sim \alpha$. Toda la gama de actitudes epistémicas entre estos dos extremos pueden ser expresadas por el hablante, $o$ asignadas a éste por la audiencia, mediante el uso de la alternancia modal. Hemos encontrado que la diferencia fundamental en las condiciones de uso que regulan tal alternancia tienen que ver con el segmento del continuo epistémico en que se mueva el hablante. El uso del indicativo está ligado a la expresión de actitudes epistémicas que van desde $\mathrm{hK} \alpha$ a $\mathrm{h} \sim \mathrm{K} \alpha$, pasando por $\mathrm{hC} \alpha, \mathrm{h} \sim \mathrm{C} \alpha$ y hC $\sim \alpha$. El uso del subjuntivo por su parte ocupa el resto del continuo epistémico, desde $h \sim K \alpha$ hasta $\mathrm{hK} \sim \alpha$, con las correspondientes actitudes intermedias $\mathbf{h} \sim \mathrm{C} \alpha$ y $\mathrm{hC} \sim \alpha$. La asignación concreta de una de estas actitudes, no obstante, no depende puramente del significado convencional de los modos, sino que está en estrecha relación con el estado del contexto que manejan el hablante y la audiencia. Teniendo en cuenta los elementos pertenecientes a tal contexto, las creencias compartidas y las atribuciones por el hablante a su audiencia y viceversa, se pueden formular principios de interpretación preferencial que predicen o regulan la asignación de una actitud epistémica $u$ otra. En particular, estos principios tienen en cuenta el caso en que la creencia está ya presente en el contexto previo a la proferencia de la oración condicional. Si hablante y audiencia comparten, por ejemplo, la creencia de que $\sim \alpha$, a figurará en modo subjuntivo en estructuras condicionales. Es más, en ciertas ocasiones la audiencia podrá introducir o considerar introducidas ciertas creencias en el contexto, en la parte de éste constituida por las creencias atribuidas al hablante, a partir de las proferencias de éste en indicativo o subjuntivo.

Nuestra explicación de los presuntos fenómenos presuposicionales ligados a las estructuras condicionales pone de relieve, desde el punto de vista pragmático, la inadecuación de generalizaciones o predicciones que ignoran el compo- 
nente contextual y el papel explicativo que tienen las intenciones comunicativas, en este caso la expresión de actitudes epistémicas, en el análisis del comportamiento lingüístico y, más específicamente, en el de la interacción comunicativa conversatoria. 


\section{REFERENCIAS BIBLIOGRAFICAS}

Bustos, E. (1982), Teorías semántica y pragmática de la presuposición, Tesis doctoral, Universidad Autónoma de Madrid.

Grice, H.P. (1968), "Utterer's meaning, sentence-meaning and wordmeaning", Foundations of Language, 4.

- (1969), "Utterer's meaning and intentions", The Philosophical Review, 78.

-. (1981), "Presupposition and conversational implicature", en P. Cole, ed., Radical Pragmatics, Nueva York, Academic Press, 1981.

Hintikka, J. (1962), Knowledge and Belief, Ithaca, Cornell U. Press. Hooper, J.B. y T. Terrell (1974), "A semantically based analysis of mood in Spanish", Hispania, 57.

Klein, F. (1975), "Pragmatics constraints on distribution: the spanish subjuntive", Chicago Linguistic Society, 11.

Lakoff, G. (1971), "Presupposition and relative well-formedness", en D. Steinberg y C. Jakobovits, eds., Semantics, Cambridge, Cambridge U. Press, 1971.

Lapesa, R. (1979), "Sobre dos tipos de subordinación causal", en Homenaje a F. Alarcos, Tomo III, Oviedo.

Lewis, D. (1973), Counterfactuals, Cambridge, Mass., Harvard U. Press.

Manteca, A. (1981), Gramática del subjuntivo, Madrid, Cátedra. Pollock, J. (1976), Subjuntive reasoning, Philosophical Studies, 8, Dordrecht, Reidel.

Stalnaker, R. (1968), "A theory of conditionals", en N. Rescher, ed., Studies on Logical Theory, Oxford, Blackwell. 
In contrast with the logico-semantical analyses of conditional (Stalnaker, 1968; Lewis, 1973; Pollock, 1976), it is claimed in this paper that it is not possible to give a sound interpretation to such sentential structures if pragmatic factors are not taken into account. In particular, determinants in the assigment of meaning to any such type of conditional are the epistemic attitude of the speaker towards the protasis, and the nature of the communicative context at the time of the utterance. On the other hand, it is argued that the inferential relations of these conditionals, have their origin in the conventional procedures which the expression of epistemic attitudes follow and which underlie all the conditional, indicative and subjunctive structures in Spanish. 